\title{
Evolutionary history of carabid ground beetles with special reference to morphological variations of the hind-wings
}

\author{
By Yûki Imura, ${ }^{* 1, \#}$ Osamu TominaGA, ${ }^{* 2, \#}$ Zhi-Hui Su,${ }^{* 3, * 4, \dagger, \#}$ Nobuo KaShiwaI, ${ }^{* 5}$ \\ Munehiro OKамото ${ }^{* 6}$ and Syozo OSAWA ${ }^{* 7, \dagger}$
}

(Communicated by Tsuneyoshi KuroiwA, M.J.A.)

\begin{abstract}
Most beetles belonging to the subfamily Carabinae of the family Carabidae (socalled carabid ground beetles) cannot fly, because their hind-wings are highly degenerated. However, about half of the species in the subtribe Calosomina within the same subfamily can fly. From extensive morphological examinations of the hind-wings of Carabinae species in conjunction with DNA molecular phylogenetic trees, the process and possible causes of hind-wing degeneration in the Carabinae are discussed.
\end{abstract}

Keywords: carabid beetle, phylogenetic tree, hind-wing degeneration

\section{Introduction}

The Carabinae is a large subfamily in the family Carabidae and is taxonomically classified into four tribes - the Cychrini, the Pamborini, the Ceroglossini, and the Carabini - based on morphological characteristics. Of these, the Carabini is further divided into two subtribes, Carabina and Calosomina. Based on the above taxonomy, we used mitochondrial and nuclear DNA sequence data to construct phylogenetic trees for approximately 2500 specimens collected from around the world from 1994 to the present. The results showed that the evolution of carabid beetles has proceeded with a combination of episodes of explosive radiation,1),2) gradual and slow species formation ${ }^{2)}$ and silent evolution, ${ }^{3)}$ which

\footnotetext{
*1 1249-8, Shinohara-Chô, Kôhoku-ku, Yokohama, Kanagawa, Japan.

*2 A321, 4-1-15, Shibatuji-Cho, Nara, Japan.

*3 JT Biohistory Research Hall, Murasaki-cho, Takatsuki, Osaka, Japan.

*4 Department of Biological Sciences, Graduate School of Science, Osaka University, Toyonaka, Osaka, Japan.

*5 Hosen-Gakuen High School, Chuo, Nakano-ku, Tokyo, Japan.

*6 Primate Research Institute, Kyoto University, Inuyama, Aichi, Japan.

*7 1003, 2-4-7 Ushita-Asahi, Higashi-Ku, Hiroshima 7320067, Japan.

$\dagger$ Correspondence should be addressed: Z.-H. Su, JT Biohistory Research Hall, 1-1 Murasaki-cho, Takatsuki, Osaka 569-1125, Japan (e-mail: su.zhihui@brh.co.jp), S. Osawa, 1003, 24-7 Ushita-Asahi, Higashi-Ku, Hiroshima 732-0067, Japan (e-mail: osawasyozo@nifty.com).

\# These authors contributed equally to this work.
}

have been characterized by the absence of morphological change over extended periods of time. ${ }^{1-3)}$ We also found that numerous hybrid-derived populations have arisen over the course of carabid evolution. ${ }^{4)}$ However, we did not pay much attention to the hind-wings of the specimens used in the above studies, because the hind-wings of carabid beetles (except for some Calosomina-species) are vestigial or absent.

The degeneration of the hind-wings in the carabid beetles is considered to have played an important role in species differentiation because the inability to fly has increased geographic isolation $\left(\right.$ e.g., Moore $\left.{ }^{5)}\right)$. This aspect is applicable to a number of beetle families with vestigial hind-wings (see Ikeda et $\left.a l .{ }^{6)}\right)$. Another aspect of this study was to understand the process and underlying reasons for the degeneration of the hind-wings. Inaizumi ${ }^{7)}$ proposed the existence of two routes for hind-wing degeneration by examining 11 Japanese Carabina species. Sugiki $^{8)}$ demonstrated that ecological conditions affect the magnitude of hind-wing degeneration in the Japanese carabid, Limnocarabus maacki. Inspired by these two works, we systematically studied the process of hind-wing degeneration and suggest that the ecological factors are responsible for hindwing degeneration phenomena. Because such investigations should be based on a reliable phylogeny, we constructed molecular phylogenetic trees for these ground beetles in conjunction with geographic events. 


\section{Materials and methods}

To clarify the evolutionary history of the subfamily Carabinae, molecular phylogenetic analyses and estimates of divergence time were performed using mitochondrial (mt) ND5 gene sequence data published in our previous studies. ${ }^{2)}$ However, the mt gene sequences selected for phylogenetic analysis were investigated using more recent and robust analytical methods, which allowed the divergence time of the Carabinae, including all tribes and subtribes, to be estimated.

The DNA sequences (1069 sites) were aligned using MAFFT, ${ }^{9)}$ and no indels were found in all alignment data sets. Phylogenetic analyses with 500 bootstrap replications were performed using MEGA7 $7^{10)}$ with the Maximum Likelihood (ML) method based on the Tamura-Nei model, ${ }^{11)}$ which is the best-fit model determined using MEGA7 with $\mathrm{BIC}$ and $\mathrm{AICc}$ criteria. The time scale involved for the tree was inferred using the RelTime method ${ }^{12)}$ and the Tamura-Nei model in MEGA7. The divergence time was computed using three calibration constraints (see Fig. 1), with the evolutionary rate of the mtND5 gene of carabid ground beetles as 3.6 million years per 0.01 pairwise Kimura-two parameter distance. ${ }^{1), 2)}$

Considering the classification based on molecular phylogenetic analyses, we examined the hindwings of 54 carabid species in 19 genera, representing all the tribes and subtribes of the subfamily Carabinae around the world (Table 1).

\section{Results}

1) Divergence time in the subfamily Carabinae estimated using molecular phylogenetic analyses. The classical taxonomic classification based on morphology of the subfamily Carabinae was described in the Introduction. The classification from the results of molecular phylogenetic analyses is generally in agreement with the classical one. However, in the classical taxonomy, the Pamborini and the Ceroglossini are treated as two independent tribes. The molecular phylogeney clearly indicates that the morphology-based Pamborini and the Ceroglossini should be unified into a single tribe in which two subtribes the Pamborina and the Ceroglossina may be settled (Fig. 1). We then propose a new classification system of the subfamily Carabinae based on the molecular phylogeny (Table 2). To discuss the degeneration pattern of the Carabinae hind-wings (see discussion section), a phylogenetic tree with expanded representative species especially focused to the Calosomina species was constructed, and this tree also supports the sister relationship between the Pamborina and the Ceroglossina (Fig. 2).

The approximate distribution ranges of all the molecular phylogeny-based tribes and subtribes and their representative species are shown in Fig. 3 (modified from Fig. 4.6 of Ref. 2).

According to $\mathrm{Su}$ et al., ${ }^{13)}$ the oldest genus in the tribe Cychrini is Scaphinotus followed by Cychropsis, and then Cychrus (Figs. 1 and 2). The origin of the latter two genera on the Eurasian continent is likely the North American Cychrini, because Scaphinotus and its related genera are not present on the Eurasian continent.

The two subtribes in the tribe Pamborini, i.e., the Pamborina and the Ceroglossina, are considered to have separated approximately 60 million years ago (Mya) (Fig. 1). According to Cox and Moore, ${ }^{14)}$ the South American, Australian and Antarctic Regions were contiguous approximately 70 Mya before separating about 50 Mya. Thus, isolation of the Ceroglossina and the Pamborina is supported by the geological record. It is reasonable to assume that the Pamborini emerged after the Cychrini in North America, before finally reaching Australia via South America.

The tribe Carabini is the largest group in the subfamily Carabinae, and is divided into two subtribes, the Carabina and the Calosomina. The former is distributed in the Holarctic Region, and the latter has an almost cosmopolitan distribution. Differentiation of the tribe Carabini is considered to have started about 52 Mya (Fig. 1). The ancestor of this tribe likely migrated from North America to the Eurasian continent, where they underwent extensive diversification.

The Carabina and the Calosomina are sister groups, as shown in Figs. 1 and 2. However, whether the divergence of these two subtribes occurred in the New World or on the Eurasian continent cannot be determined from the phylogenetic tree alone. However, because the Carabina subtribe on the Eurasian continent is relatively species rich and only a small number of species are distributed in the New World, we assumed that divergence of these two subtribes occurred on the Eurasian continent. The species in the New World could thus be fairly recent immigrants from the Eurasian continent.

2) Hind-wing degeneration of representative species of the Carabinae. To demonstrate the 


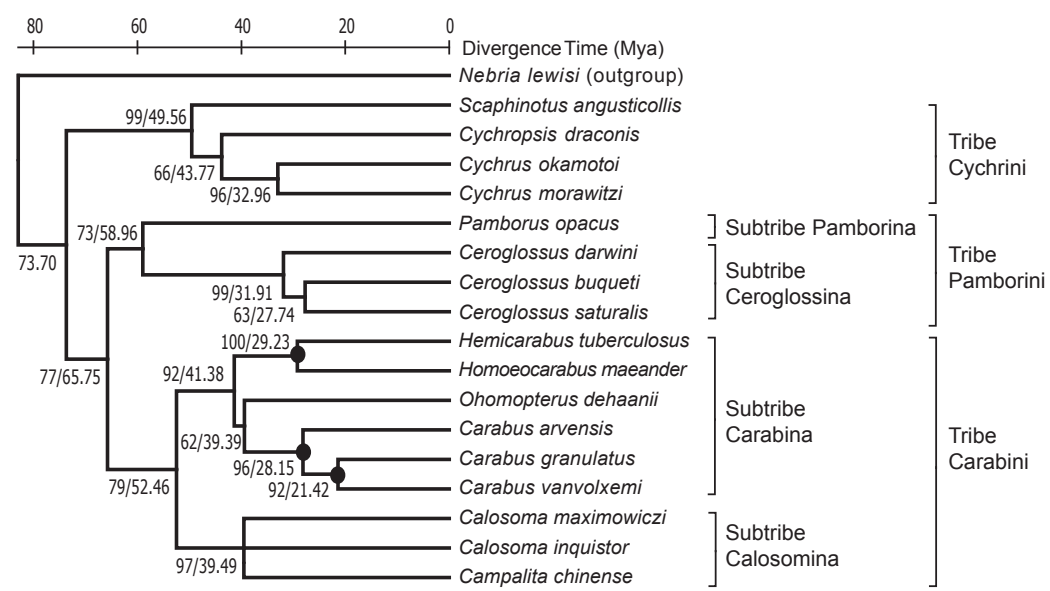

Fig. 1. Phylogenetic tree of the ND5 gene showing the chronology of the Carabinae ground beetles. The sequence data of 17 species representing the (sub)tribes were used to construct the tree. Nebria lewisi, a species of the subfamily Nebriinae, which is related to the Carabinae, was used as outgroup. Black spots indicate the calibration constraints, which are calculated with the evolutionary rate of ND5 gene of carabid beetles (see Materials and methods). The bootstrap values and computed divergence time are shown on each node separated by a slash mark.

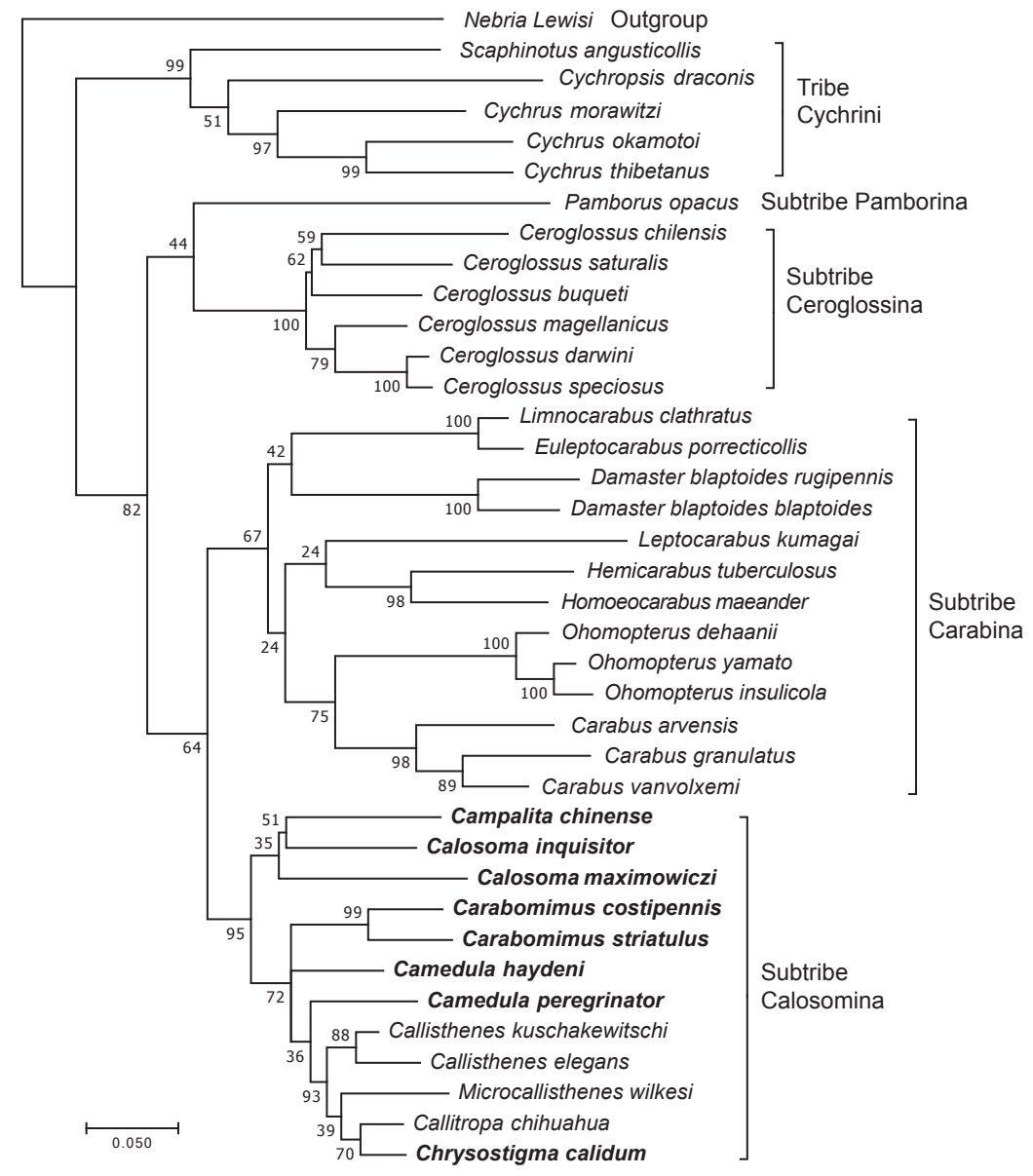

Fig. 2. Phylogenetic tree of the ND5 gene with emphasis of 37 representative species of the Carabinae ground beetles, especially focused on the Calosomina species. The bootstrap values are indicated on each node. The Calosomina species shown in bold letters have well-developed hind-wings and are capable of flight. 
Table 1. Specimens used for the examination of hind-wing degeneration

\begin{tabular}{|c|c|c|c|}
\hline Species name & Locality & Species name & Locality \\
\hline Cychrini & & Carabini (Carabina) & \\
\hline Cychrus morawitzi morawitzi & Japan: Hokkaido & Limnocarabus maacki aquatilis & Japan: Aomori, Yamagata \\
\hline C. m. iwatensis & Japan: Iwate & Euleptocarabus porrecticollis & Japan: Gifu, Niigata \\
\hline C. bispinosus bispinosus & China: Shaanxi & Hemicarabus tuberculosus & Japan: Izu-oshima, Rishiri-to \\
\hline C. minshanicola minshanicola & China: Sichuan & H. macleayi amanoi & Japan: Is. Rishiri-to \\
\hline C. thibetanus thibetanus & China: Sichuan & Homoeocarabus maeander paludis & Japan: Hokkaido \\
\hline C. koiwayai koiwayai & China: Sichuan & Asthenocarabus opaculus opaculus & Japan: Hokkaido \\
\hline C. stoetzneri stoetzneri & China: Sichuan & Pentacarabus harmandi harmandi & Japan: Tochigi \\
\hline C. furumii minshanicus & China: Sichuan & Aulonocarabus kurilensis rishiriensis & Japan: Is. Rishiri-to \\
\hline C. kaznakovi kaznakovi & China: Gansu & Leptocarabus procerulus procerulus & Japan: Yamanashi \\
\hline C. chareti chareti & China: Yunnan & L. kumagaii nishi & Japan: Osaka \\
\hline C. kralianus & China: Yunnan & Carabus arvensis hokkaidensis & Japan: Hokkaido \\
\hline C. caraboides ssp. & Russia: Polar Ural & C. granulatus telluris & Japan: Yamagata, Tochigi \\
\hline C. aeneus aeneus & Georgia: Caucasus & C. g. yezoensis & Japan: Hokkaido \\
\hline C. aeneus aeneus & Russia: Caucasus & C. vanvolxemi vanvolxemi & Japan: Nagano \\
\hline C. anatolicus ponticus & Turkey: Ordu & Ohomopterus yamato kinkimontanus & Japan: Osaka \\
\hline C. tuberculatus & USA: Washington & O. japonicus japonicus & Japan: Ehime \\
\hline Cychropsis draconis ssp. & China: Sichuan & O. dehaanii dehaanii & Japan: Osaka \\
\hline Scaphinotus (Brennus) marginatus & USA: Washington & O. insulicola insulicola & Japan: Yamanashi \\
\hline Sc. (Irichroa) viduus & USA: Washington & Megodontus kolbei kolbei & Japan: Hokkaido \\
\hline Sc. (Steniridia) andrewsii mutabilis & USA: Pennsylvania & Acoptolabrus gehinii aereicollis & Japan: Hokkaido \\
\hline Sc. (Neocychrus) behrensi behrensi & USA: California & Damaster blaptoides rugipennis & Japan: Hokkaido \\
\hline Sc. (Stenocantharus) velutinus & USA: California & D. b. blaptoides & Japan: Kumamoto \\
\hline Sphaeroderus stenostomus stenostomus & USA: Pennsylvania & Coptolabrus. fruhstorferi & Japan: Iss. Tsushima \\
\hline \multirow[t]{2}{*}{ Sp. s. lecontei } & USA: West Virginia & & \\
\hline & & Carabini (Calosomina) & \\
\hline Pamborini (Pamborina) & & Campalita chinense & Japan: Hiroshima \\
\hline Pamborus alternans & Australia: Queensland & Calosoma inquisitor cyanescens & Japan: Hokkaido \\
\hline \multirow[t]{2}{*}{ P. opacus } & Australia: Queensland & C. maximowiczi & Japan: Hokkaido \\
\hline & & Callisthenes kuschakewitschi & Kazakhstan: Talasskij Alatau \\
\hline Pamborini (Ceroglossina) & & Carabops burtoni & Tanzania, Mbeya Region \\
\hline Ceroglossus chilensis chilensis & Chile: Concepción & Chrysostigma calidum & USA: Michigan \\
\hline C. buqueti buqueti & Chile: La Union & & \\
\hline C. magellanicus magellanicus & Chile: Mariquina & & \\
\hline C. speciosus speciosus & Chile: Is. Chiloé & & \\
\hline
\end{tabular}

Table 2. Classification of the subfamily Carabinae

\begin{tabular}{cc}
\hline Generally used classification & Classification proposed in this study $\left.{ }^{*}\right)$ \\
\hline Subfamily Carabinae Latreille, 1802 & Subfamily Carabinae Latreille, 1802 \\
1. Tribe Cychrini Laporte, 1834 & 1. Tribe Cychrini Laporte, 1834 \\
2. Tribe Pamborini Lacordaire 1854 & 2. Tribe Pamborini Lacordaire 1854 \\
3. Tribe Ceroglossini Lapouge, 1927 & 1) Subtribe Pamborina Lacordaire 1854 \\
4. Tribe Carabini Latreille, 1802 & 2) Subtribe Ceroglossina Lapouge, 1927 \\
1) Subtribe Carabina Lapouge, 1927 & 3. Tribe Carabini Latreille, 1802 \\
2) Subtribe Calosomina Lapouge, 1927 & 1) Subtribe Carabina Lapouge, 1927 \\
& 2) Subtribe Calosomina**) Lapouge, 1927 \\
\hline
\end{tabular}

${ }^{*}$ The purpose of this study was not to recommend nomenclatural changes but to contribute phylogeny and evolutionary history of the carabid beetles. Therefore, we use these names for the sake of convenience.

${ }^{* *}$ According to the International Code of Zoological Nomenclature 1999, Art. 29.3.1, the spelling of this subtribe name should be Calosomatina, but we adopt currently spelled Calosomina according to Art. 29.5. 

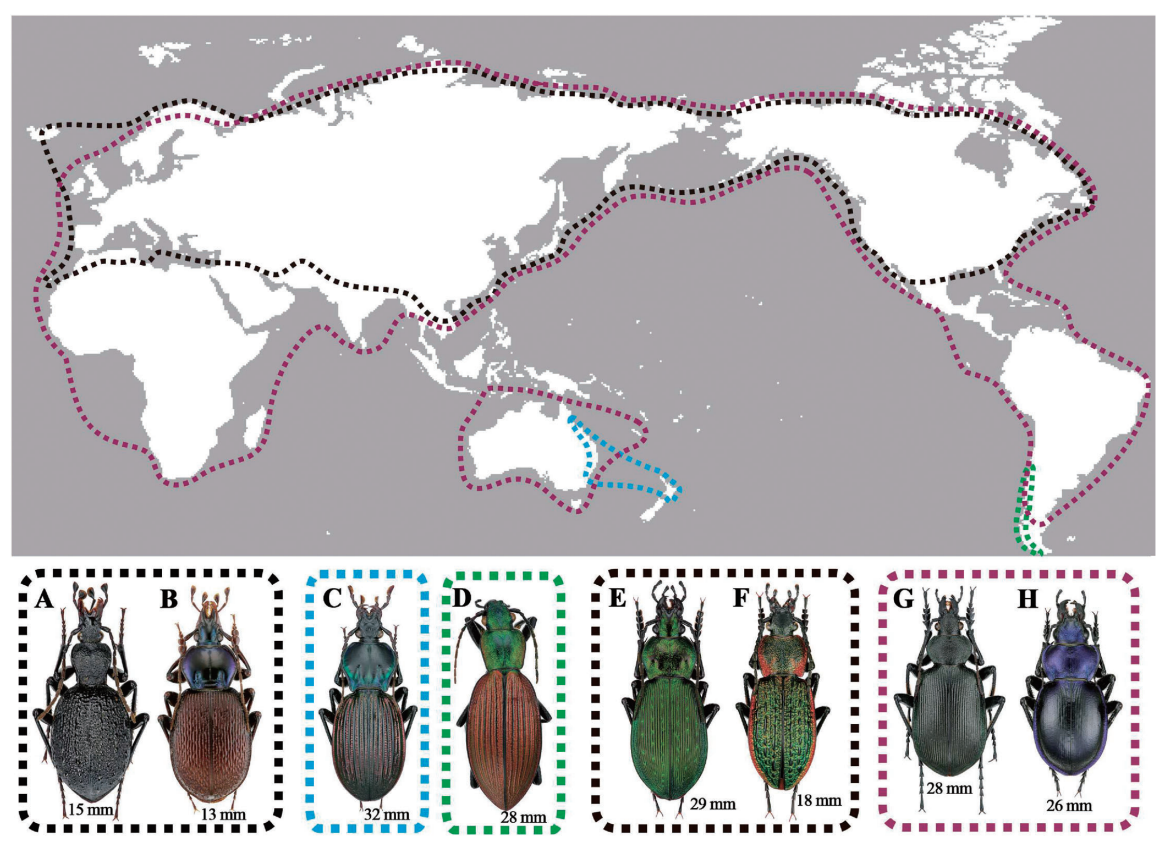

Fig. 3. Map showing the approximate distribution ranges of all the tribes and subtribes of the subfamily Carabinae in the world. A black dotted line indicates the ranges of the Cychrini containing nearly 180 species $^{20)}$ and the Carabina containing more than 900 species, ${ }^{21)}$ a blue line indicates that of the Pamborina containing 17 species, a green line indicates that of the Ceroglossina (containing 8 species), and a purple line indicates that of the Calosomina containing nearly 130 species. ${ }^{18)}$ Representative species of each tribe and subtribe are shown at the bottom (A, Cychrus morawitzi (Cychrini); B, Scaphinotus marginalis (ditto); C, Pamborus alternans (Pamborina); D, Ceroglossus chilensis (Ceroglossina); E, Ohomopterus insulicola (Carabina); F, Hemicarabus macleayi (ditto); G, Calosoma maximowiczi (Calosomina); H, Callisthenes kuschakewitschi (ditto)).
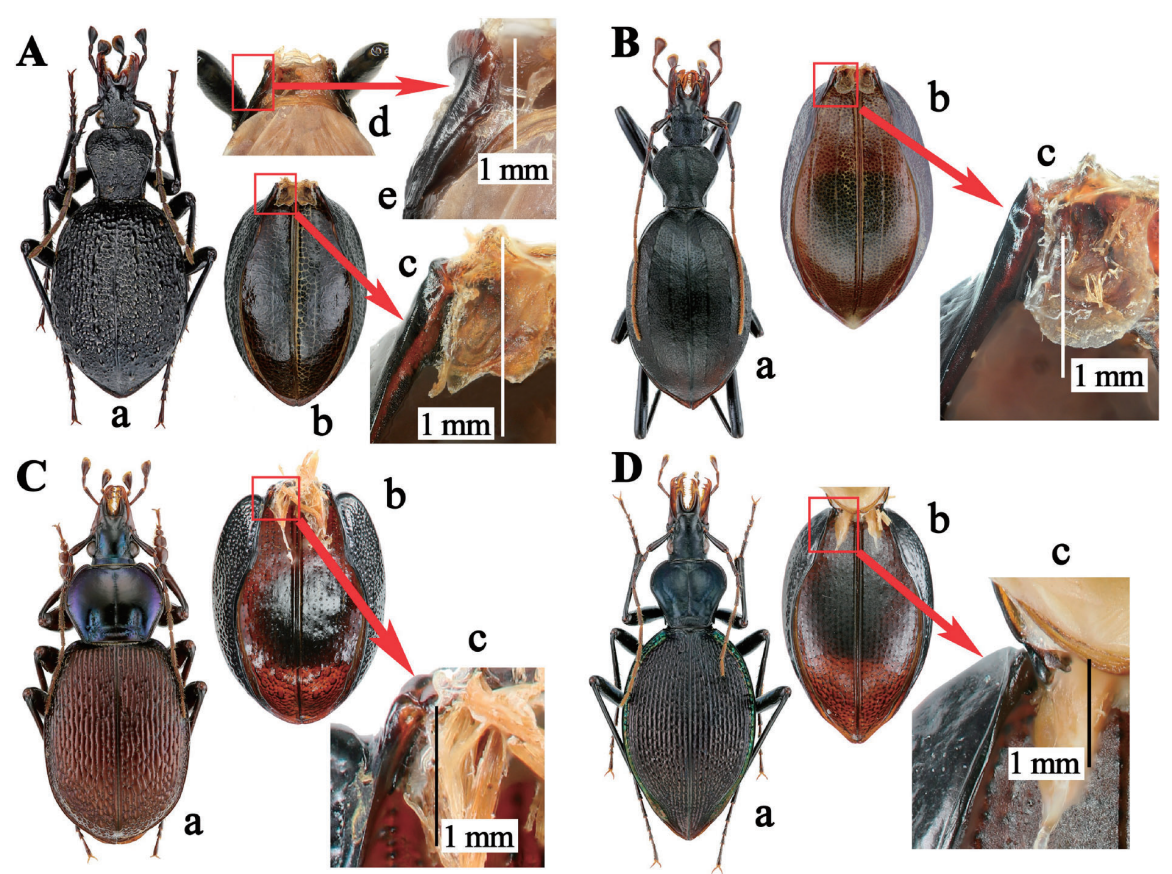

Fig. 4. Four species of the tribe Cychrini.-A, Cychrus morawitzi (Iwate, Japan); B, Cychropsis draconis (Sichuan, China); C, Scaphinotus (Brennus) marginatus (Washington, USA); D, Sphaeroderus stenostomus (Pennsylvania, USA). —a, habitus in dorsal view; b, elytra (removed from abdomen) in ventral view; c, magnified view of right elytron (basal part) in ventral view; d, basal part of abdomen in dorsal view (elytra were removed); e, magnified view of the left abdomen in dorsal view. Hind-wings cannot be recognized. 
patterns of wing degeneration in the Carabinae, we illustrated 11 representative species belonging to all of the (sub)tribes of the subfamily Carabinae (Figs. 4-6). In principle, the hind-wing is composed of the flight muscles and a membranous wing. In most Carabinae species, the membranous wings are typically vestigial (e.g., see Figs. 5A-d and 5B-d, where the flight muscle and degenerated wing are shown by blue and green arrows, respectively). However, in 5 species of 4 genera of the Cychrini from Eurasia and North America, their hind wings and flight muscles cannot be recognized, indicating that the hind-wings of the Cychrini are the most vestigial among all of the tribes in the Carabinae (Fig. 4). Indeed, the hind-wings and the associated flight muscles were totally absent in all of the Cychrini. In the tribe Pamborini (comprised of the Pamborina and the Ceroglossina) and the Carabini (comprised of the Carabina and the Calosomina), the hind-wings of the Pamborini and the Carabina are more or less degenerated, whereas about half of the species belonging to the Calosomina are winged.

The hind-wing of Pamborus alternans (the subtribe Pamborina) is remarkably atrophic (Fig. 5A), forming a needle-like narrow process tightly attached to the inner basal portion of the elytral epipleuron via the flight muscle (Fig. 5A-d). The degree of hind-wing degeneration in Ceroglossus chilensis (Fig. 5B-d) is similar to that observed in Pamborus spp. The pattern of hind-wing degeneration in other species of the subtribes Pamborina and Ceroglossina is essentially similar to that of $P$. alternans we have examined to date (see Table 1).

The hind-wings of Carabina spp. show varying degrees of degeneration. In both Hemicarabus macleayi (Fig. 5C) and Ohomopterus insulicola (Fig. 5D), the hind-wings are highly degenerated, although the pattern of atrophy in these species is unequal and equal, respectively (see Section 3).

Figure 6A shows Calosoma maximowiczi (the subtribe Calosomina, the tribe Carabini) found in woodland habitants. This species has hind-wings that are well-developed and can be used for flight. Figures $6 \mathrm{~B}$ and $6 \mathrm{C}$ show Callisthenes kuschakewitschi and Carabops burtoni (the subtribe Calosomina, the tribe Carabini), which are found in arid regions; the hind-wings of these species are highly degenerated and probably cannot be used for flight. In the phylogenetic tree (Fig. 2), the flyable and unflyable Calosomina species (respectively shown by bold and regular letters in Fig. 2) do not form independent lineage. Although we examined only two species having degenerated hind-wings in this study, the other species shown by regular letters in Fig. 2 also have degenerated hind-wings (see pp. 43-44 of Ref. 2).

3) Degeneration pattern of the hind-wings within the Japanese Carabinae. Figure 7 shows left hind-wing of 17 representative species of the Carabina occurring in Japan. Inaizumi ${ }^{7)}$ recognized two types of wing degeneration, namely, equal degeneration-type corresponding to Fig. 7E-7I, and unequal degeneration-type (Fig. 7M-7R). However, it is difficult to accept Inaizumi's scheme of two distinct types of wing degeneration because several species show an intermediate degeneration-type (Fig. 7J-7L). In certain species, such as Limnocarabus maacki, Carabus granulatus and C. arvensis, there exist individuals having both well-developed hind-wings (Fig. 8A-a, 8B-a, 8C-a) and highly degenerated hind-wings (Fig. 8A-b, 8B-b, 8C-b). In these three species, there exist individuals having intermediary sized hind-wings (see Discussion 3).

We then propose an apparently continuous degeneration profile for the Japanese Carabina species.

\section{Discussion}

1) Generalized view of hind-wing degeneration based on the phylogenetic trees. As shown in the molecular phylogenetic trees (Fig. 1, see also Fig. 4.5 of Osawa et al. ${ }^{2)}$ ) the subfamily Carabinae originated from non-Carabinae ground beetles about 80 Mya. The oldest tribe in the Carabinae is the Cychrini, followed by the tribe Pamborini (which contains the subtribes Pamborina and the Ceroglossina) and finally the tribe Carabini (which contains the subtribes Carabina and Calosomina). The hindwings of all the Carabinae beetles, except for some Calosomina, are either absent or degenerated, and the degree of degeneration macroscopically matches the above-mentioned phylogenetic order at the (sub)tribe level.

Because hind-wing degeneration in the Cychrini and the Pamborini is at the end or nearly the end stages, the detailed process of hind-wing degeneration cannot be traced. However, hind-wing degeneration of different stages can be observed in the Carabina species, in which the extent of hind-wing degeneration does not reflect the phylogenetic age (see Figs. 1, 2, 7 and also Section 3 of the Discussion).

In addition, all of the members of the Carabinae possess forewings (elytra), whereas their hind-wings are either vestigial or absent (except for some 

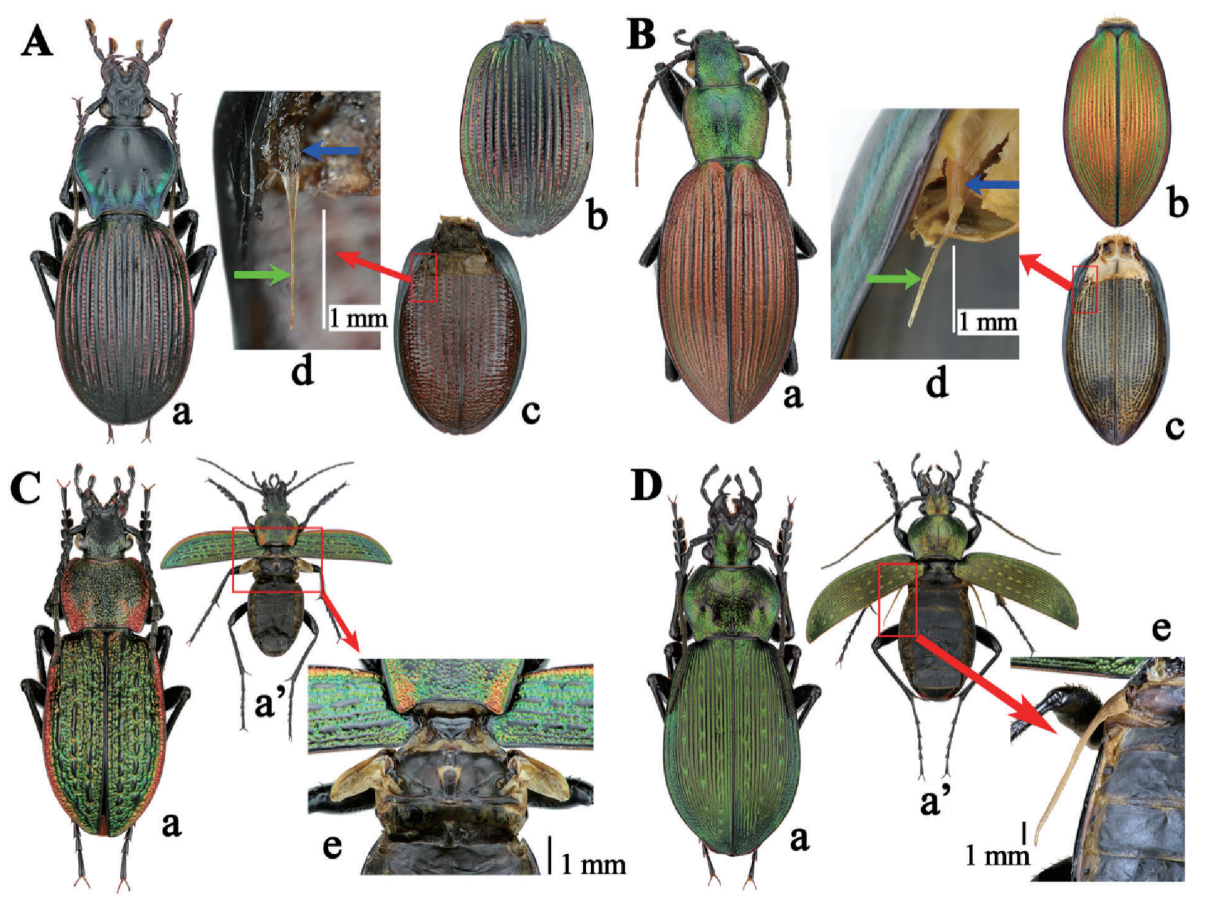

Fig. 5. Four species of the tribes Pamborini and Carabini._A, Pamborus alternans (New South Wales, Australia); B, Ceroglossus chilensis (Concepción, Chile); C, Hemicarabus macleayi (Is. Rishiri-to, Japan); D, Ohomopterus insulicola (Kanagawa, Japan). a, habitus in dorsal view; $\mathrm{a}^{\prime}$, ditto (elytra were opened); b, elytra in dorsal view; c, ditto in ventral view; d, needle-like, vestigial right hind-wing adhered to apical inner portion of elytral epipleuron; e, degenerated left hind-wing. In A-d and B-d, a blue arrow indicates flight muscle and a green arrow indicates the degenerated membranous part of hind wing.
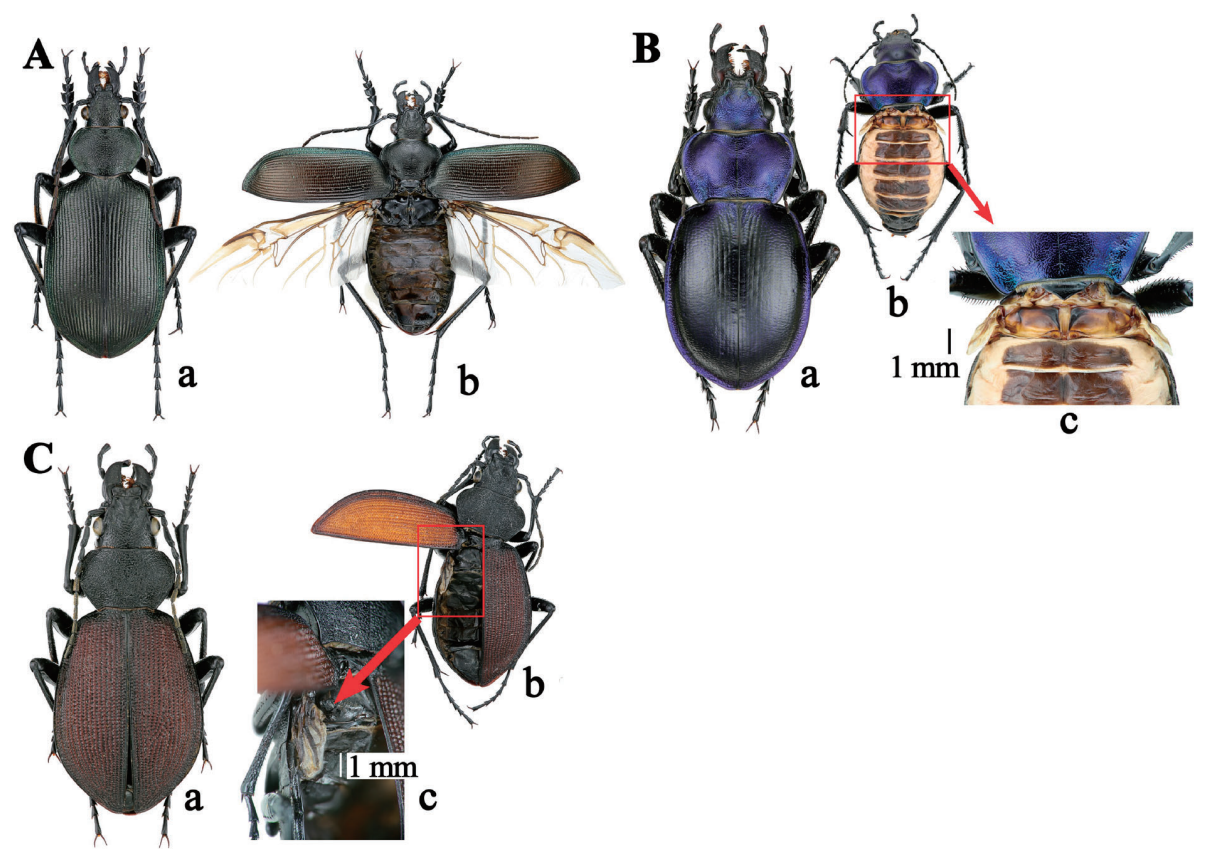

Fig. 6. Three species of the subtribe Calosomina. - A, Calosoma maximowiczi (Hokkaido, Japan); B, Callisthenes kuschakewitschi (Talasskij Alatau, Kazakhstan); C, Carabops burtoni (Mbeya Region, Tanzania). - a, habitus in dorsal view; b, ditto (elytra were opened or removed); c, magnified view of the degenerated left hind-wing. The molecular pylogenetic analysis of Carabops burtoni has not been done. 


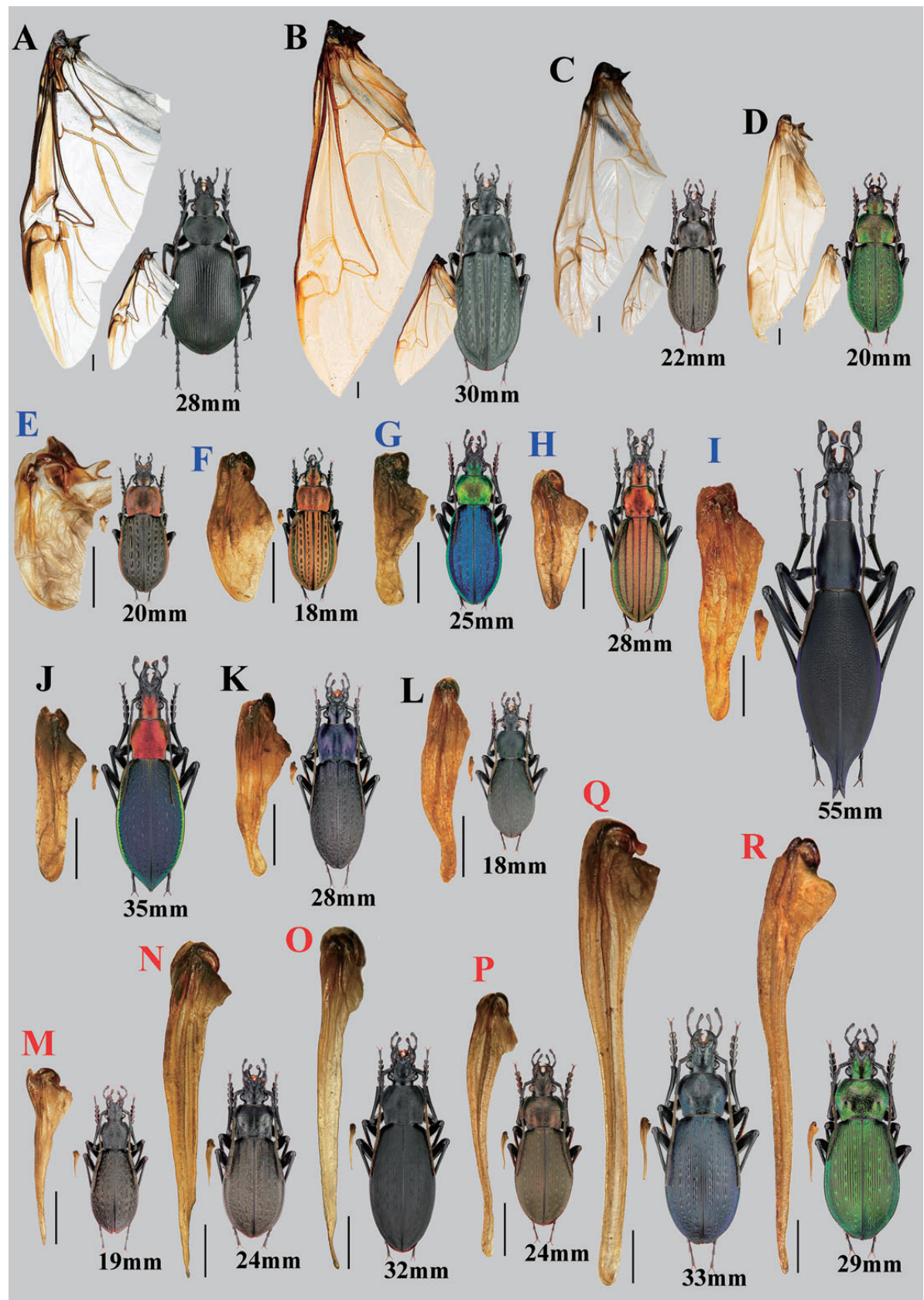

Fig. 7. Eighteen representative species of the tribe Carabini inhabiting Japan and their left hind-wings. — A, Calosoma maximowiczi; B, Limnocarabus maacki; C, Carabus granulatus; D, C. arvensis; E, Hemicarabus tuberculosus; F, Homoeocarabus maeander; G, Megodontus kolbei; H, Acoptolabrus gehinii; I, Damaster blaptoides; J, Coptolabrus fruhstorferi; K, Euleptocarabus porrecticollis; L, Asthenocarabus opaculus; M, Pentacarabus harmandii; N, Aulonocarabus kurilensis; O, Leptocarabus procerulus; P, Ohomopterus japonicus; Q, O. dehaanii; R, O. insulicola. Right: habitus; middle: left hind-wing shown in the same reduction ratio as that of the habitus; left: ditto in magnified view. Scale bar: $1 \mathrm{~mm}$ for magnified view of left hind-wing. The blue and red letters indicate the hind-wing of the equal atrophy degeneration type and unequally atrophy degeneration type of Inaizumi, respectively. ${ }^{7}$

Calosomina spp.). These findings strongly suggest that the development of the fore- and hind-wings are under different control mechanisms.
2) The mother lineage of the Carabinae as deduced from hind-wing degeneration patterns. The ancestor of the Carabinae was likely a flyable 


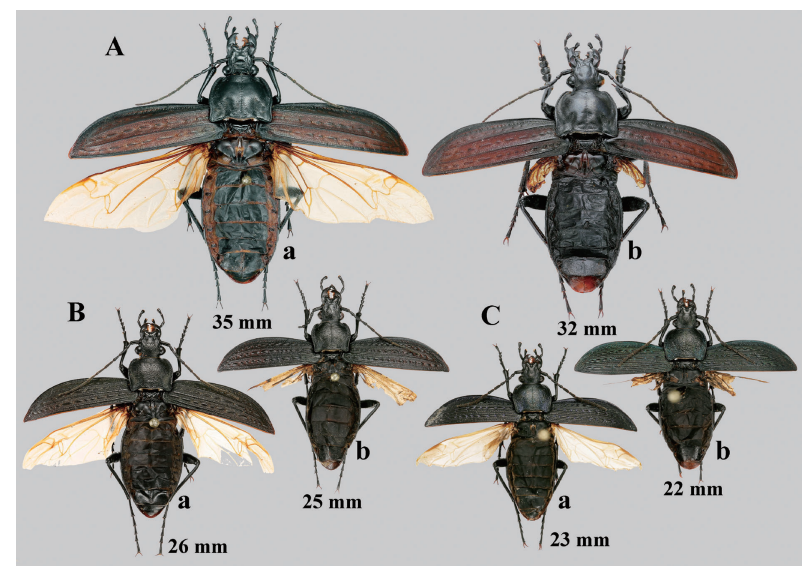

Fig. 8. Individual variation of the hind-wings of three Carabina species inhabiting Japan.—A, Limnocarabus maacki (a, from Yamagata; b, from Aomori); B, Carabus granulatus (a, from Tochigi; b, from Hokkaido); C, C. arvensis (both a and b from Hokkaido); a, individual with well-developed hind-wings; b, individual with degenerated hind-wings.

and, carnivorous, non-carabinae, ground beetle that inhabited forests. Among the extant (sub)tribes, such habitat preference is seen in the subtribe Calosomina. Thus, it seems likely that the parental lineage could have started from the ancestral Carabinae and extended to the extant subtribe Calosomina. The Cychrini, the Pamborini, and the Carabini are thus tribes that branched off from the mother lineage. In other words, the ancestral form of the Carabinae could have well developed hind-wings that were capable of flight. Figure 9 shows a hypothetical evolutionary tree with the Cychrini, the Pamborini, and the Carabina branching from the mother lineage.

3) Factors affecting degeneration of the hindwings. It is difficult to speculate how and why the hind-wings began to degenerate from the winged ancestor of the Carabinae. In the case of the Cychrini, it may have been easier to obtain food, such as earthworms, insect larvae, and land snails on and below the ground surface (see Lindroth ${ }^{15)}$ ) than to catch small insects in the trees. Consequently, the Cychrini became adapted to ground environments. Given the ancestral form, the Cychrini no longer had to fly to catch its prey, the hind-wings became unnecessary and disappeared.

The tribe Pamborini could have branched off from the mother lineage in the barren areas of South America, where there are no trees and the Pamborini species were forced to hunt for prey on land. Thus, their hind-wings became unnecessary and degenerated.

The morphology and molecular phylogenetic tree of the Carabina provide us with several clues regarding the degeneration of the hind-wings, which occurred after branching off from the parental (the Calosomina) lineage. The extent of hind-wing degeneration does not reflect phylogenetic age. For example, although $C$. granulatus and $C$. arvensis are very old species, they have relatively moderate-sized wings; conversely, while Damaster blaptoides and Ohomopterus spp. emerged much later than Carabus spp., these species have highly degenerated hind-wings (for a molecular phyloge-

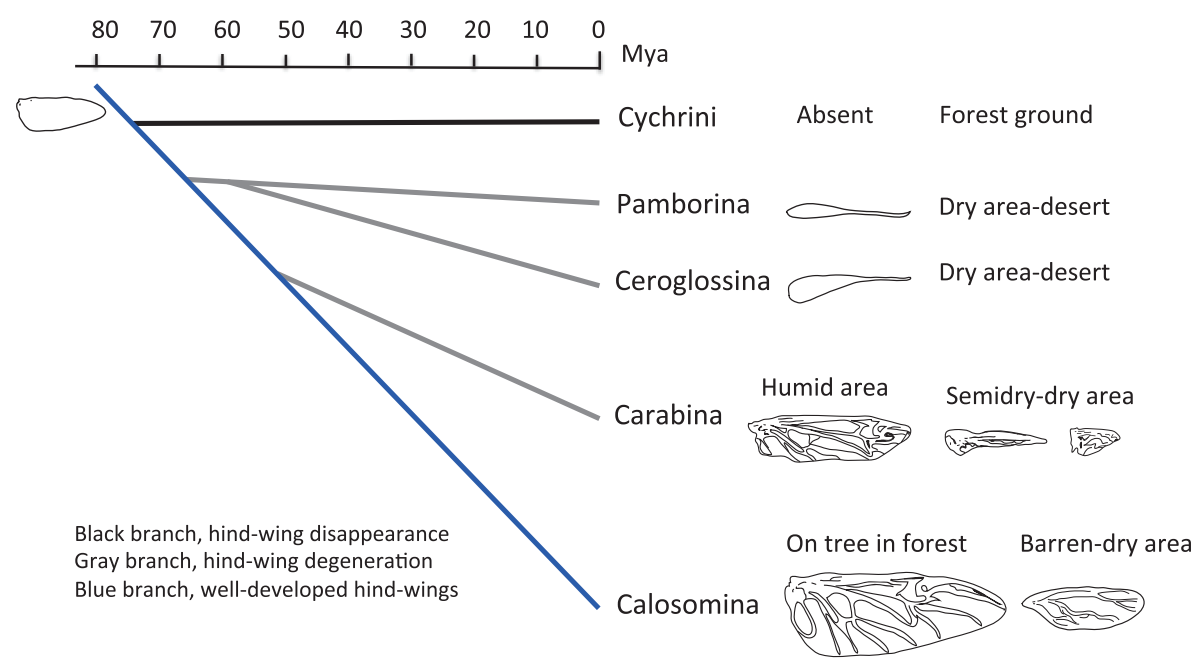

Fig. 9. Simplified molecular phylogenetic tree of the subfamily Carabinae showing the process of hind-wing degeneration. Estimated divergence times are according to Fig. 1. Illustrations of the hind-wings of some representative species are shown schematically on the right side. On the left side of the Cychrini line shows the hind-wing of the hypothetical ancestral Carabinae species. 
netic tree of the Japanese Carabina, see Fig. 6.3 in Osawa et $a l_{.}^{2)}$ ).

As discussed below, we consider that the prevailing ecological conditions act as a trigger for hind-wing degeneration rather than the phylogenetic age. The most remarkable examples of the effect of ecological conditions may be seen in the individuals with relatively well conserved (Fig. 8A-a) and highly degenerated (Fig. 8A-b) hind-wings of L. maacki, C. granulatus (Fig. 8B), or C. arvensis (Fig. 8C). These figures seem to show the effect of a simple mutation on hind-wing dimorphism in each species. However, Sugiki ${ }^{8}$ examined about 50 L. maacki specimens from the area surrounding Lake Jûsan-ko in Aomori Prefecture in northern Japan and found individuals with variously sized hind-wings. Notably, however, individuals with relatively well conserved hind-wings tended to occur more frequently in the areas close to the lake. C. granulatus is an example of the possible effect of humidity. The population of C. granulatus occurring in Hokkaido, Japan, is stenotopic and hygrophilous, with most individuals in a population having hind-wings that are relatively well conserved (Fig. 8B-a). Conversely, the populations in Honshu are eurytopic with hind-wings that are typically more degenerated than the Hokkaido population (Fig. 8B-b). In addition, many individuals with intermediate-sized hind-wings are recognized in both populations. The hind-wings of $C$. granulatus from North America are degenerated and somewhat variable in size; no individuals capable of flight have been observed to date. ${ }^{15}$ ) In other words, hind-wing degeneration proceeds gradually and cannot be explained by a simple dimorphic genetic mutation. The extent of hind-wing degeneration in hygrophilous species such as Hygrocarabus nodulosus, $H$. variolosus, and other species is much less pronounced than that in the same species found in drier areas. ${ }^{16), 17)}$

The predominance of individuals with relatively well-developed hind-wings in wetlands - compared with individuals with highly degenerated hind-wings in arid areas - favors the hypothesis that the surrounding ecological conditions (in this case, humidity) may act as a trigger for hind-wing degeneration. However, humidity is not likely to be the only ecological factor affecting hind-wing degeneration.

Nearly half of the extant Calosomina spp. can fly (Fig. 6A), but the remaining half which inhabit arid areas are flightless (see Bruschi ${ }^{18)}$ ). The Calosomina species with well-developed hind-wings inhabit for- ested areas and are generally arboreal predators of caterpillars.

A Calosomina species Callisthenes kuschakewitschi from arid areas of the Talasskij Alatau in Kazakhstan (Fig. 6B) has highly degenerated hindwings. The situation could be similar for other Calosomina species with degenerated hind-wings, such as species inhabiting arid regions or high altitudes, including those found in central Asia, parts of Africa, and western North America. The above observations suggest that once the ecological conditions changed and woodlands became arid areas and caterpillars on trees were unavailable, the Calosomina species either died out or survived on the land surface. The hind-wings of species inhabiting these environments became unnecessary and degenerated as in the Carabina species. These flightless, brachypterous species are forced to prey on the larvae of various small arthropods or other soil-dwelling organisms. It is highly plausible that the capacity for flight in brachypterous species is linked to their ecological (feeding) habitat. Of note, several flightless Calosomina species are grouped with flying Calosomina species and do not form an independent branch on the phylogenetic tree (see Fig. 2). Therefore, this observation suggests that degeneration of the hindwings is controlled by ecological conditions regardless of phylogenetic lineages.

In summary, we propose that (1) the rate of hind-wing degeneration depends on the surrounding ecological conditions and not on phylogenetic age, at least in the subtribe Carabina, and (2) that hindwing degeneration probably occurs irreversibly. See also Fig. 9 for a simplified molecular phylogenetic tree of the subfamily Carabinae showing the process of hind-wing degeneration. The hind-wings of some representative species are illustrated schematically on the right side with description of inhabiting environments. On the left side of the Cychrini line shows the hind-wing of the hypothetical ancestral Carabinae species.

4) Degeneration of the hind-wings in the Coleopterous world. There exist a number of beetles whose hind-wings are highly degenerated in both sexes as in the Carabinae ground beetles discussed in this paper. These beetles can be found in the Cerambycidae, the Tenebrioidae, the Elateridae, the Curculionidae, etc. Their living territories are restricted to relatively small areas where feeding and reproductive activities are satisfied without flying activity. Thus, the hind-wings became unnecessary and degenerated. The degenerations may 
be the results of adaptation to their environment. The degeneration of this kind is a phenomenon of mere removal of "disuse" structure and thus supports the disuse part of the Lamarck's Use and Disuse Theory.

It is well known that in some lampyrid beetles, the hind-wings are either incomplete or absent in the female, and yet the male has well-developed hindwings. In this case, it is considered that incompleteness or absence of hind-wings in the female is not a result of degeneration but is due to contribution to sufficient maturation of the female reproductive organ (e.g., South et al. $\left.{ }^{19)}\right)$. This case should be distinguished from "degeneration".

\section{Conclusion}

Molecular phylogenetic analyses of the Carabidae beetles indicated that the ancestor of the socalled ground beetles (the subfamily Carabinae) could have branched off from the non-Carabinae beetles of the family Carabidae with flight capable hind-wings about 80 Mya. The mother lineage of the Carabinae started from the above non-Carabinae beetles and reached the present-day subtribe Calosomina, in which many have well-developed hindwings. During evolution, two tribes and one subtribe branched off from the mother lineage. These are (1) the Cychrini, in which the hind-wings are absent, (2) the Pamborini, with extremely degenerated hindwings, and (3) the subtribe Carabina, which is composed of many species with hind-wings in various degeneration stages. The members of 1 to 3 are not able to fly. The extent of degeneration is not phylogenetic age-dependent and seems to be largely influenced by ecological conditions, such as humidity. In the Calosomina species living in barren areas, the hind-wings are highly degenerated. This also suggested that ecological conditions are an important factor for hind-wing degeneration. As mentioned above, many Carabinae beetles do not fly because of the adaptation to live on ground. Thus, the hindwings became unnecessary and degenerated. This phenomenon may be explained in terms of removal of "a disused" structure.

\section{Acknowledgments}

We express our appreciation to Igor Belousov (St. Petersburg, Russia), Eric Van Den Berghe (Seattle, USA), Robert Davidson (Carnegie Institution, Pittsburgh, USA), Ilya Kabak (St. Petersburg, Russia), Robert Lizler (Hradec Králové, Czech Republic), Yoshiyuki Nagahata (Yamagata, Japan),
Yutaka Nishijima (Hokkaido, Japan), Yuichi Oba (Nagoya, Japan) and Masahiko Sato (Hokkaido, Japan) for useful comments or supplying invaluable specimens. Thanks are also due to Ayako Sasaki (JT Biohistory Research Hall, Osaka, Japan) for drawing the figures of the hind-wings shown in Fig. 9.

\section{References}

1) Su, Z.-H., Imura, Y. and Osawa, S. (2001) Evolutionary discontinuity of the carabine ground beetles. J. Mol. Evol. 53, 517-529.

2) Osawa, S., Su, Z.-H. and Imura, Y. (2004) Molecular Phylogeny and Evolution of Carabid Ground Beetles, Springer-Verlag, Tokyo.

3) Osawa, S., Su, Z.-H., Nishikawa, M. and Tominaga, O. (2016) Silent evolution. Proc. Jpn. Acad. Ser. B 92, 455-461.

4) Su, Z.-H., Okamoto, M., Tominaga, O., Akita, K., Kashiwai, N., Imura, Y. et al. (2006) Establishment of hybrid-derived offspring populations in the Ohomopterus ground beetles through unidirectional hybridization. Proc. Jpn. Acad. Ser. B 82, 232-250.

5) Moore, B.P. (1966) The larva of Pamborus (Coleoptera: Carabidae) and its systematic position. Proc. R. Ent. Soc. Lond. B 35, 1-4.

6) Ikeda, H., Nishikawa, M. and Sota, T. (2012) Loss of flight promotes beetle diversification. Nat. Commun. 3, 648 .

7) Inaizumi, M. (1966) Studies on the hind-wing morphology of the Carabinae in Japan (Coleoptera). Kontyû 34, 248-265 (in Japanese with English summary).

8) Sugiki, T. (1986) On the hind-wings of Apotomopterus (Limnocarabus) clathlatus maacki (sic) (Morawitz) from the surrounding area of the lake Jûsan-ko, Aomori Prefecture. Aomori no chô 9, 38-42 (in Japanese).

9) Katoh, K., Kuma, K., Toh, H. and Miyata, T. (2005) MAFFT version 5: Improvement in accuracy of multiple sequence alignment. Nucleic Acids Res. 33, 511-518.

10) Kumar, S., Stecher, G. and Tamura, K. (2016) MEGA7: Molecular Evolutionary Genetics Analysis version 7.0 for bigger datasets. Mol. Biol. Evol. 33, $1870-1874$.

11) Tamura, K. and Nei, M. (1993) Estimation of the number of nucleotide substitutions in the control region of mitochondrial DNA in humans and chimpanzees. Mol. Biol. Evol. 10, 512-526.

12) Tamura, K., Battistuzzi, F.U., Billing-Ross, P., Murillo, O., Filipski, A. and Kumar, S. (2012) Estimating divergence times in large molecular phylogenies. Proc. Natl. Acad. Sci. U.S.A. 109, 19333-19338.

13) Su, Z.-H., Imura, Y., Okamoto, M. and Osawa, S. (2004) Pattern of phylogenetic diversification of the Cychrini ground beetles in the world as deduced mainly from sequence comparisons of the mitochondrial genes. Gene 326, 43-57. 
14) Cox, C.B. and Moore, P.D. (1980) Biogeography: An Ecological and Evolutionary Approach. 3rd Edition, Blackwell Scientific Publications, Oxford.

15) Lindroth, C.H. (1961) The ground-beetles (Carabidae excl. Cicindelinae) of Canada and Alaska. Part 2 Opuscula Entomologica Supplementum 20, pp. 1-200. Lund.

16) Imura, Y. and Mizusawa, K. (1996) The Carabus of the World, Mushi-sha, Tokyo.

17) Casale, A., Sturani, M. and Vigna Taglianti, A. (1982) Coleoptera: Carabidae. I. Introduzione, Paussinae, Carabinae. Fauna d'Italia, 18, XII + 499pp., Calderini, Bologna.

18) Bruschi, S. (2013) Calosoma of the World, Natura Edizioni Scientifiche di Alfonso Iorio, Bologna.
19) South, A., Sthanger-Hall, K., Lung, M.-L., Jeng, M.-L. and Lewis, S.M. (2011) Correlated evolution of female neoteny and flightlessness with male spermatophore production in fireflies (Coleoptera: Lampyridae). Evoluton 65, 1099-1113.

20) Cavazzuti, P. (2010) World catalogue of the tribe Cychrini (Coleoptera, Carabidae, Carabinae). Annali del Museo Civico di Storia Naturale "G. Doria" 102, 203-294.

21) Deuve, Th. (2016) Classification du Genre Carabus L., 1758. Liste Blumenthal 2016-2017, Association Magellanes, Andrésy.

(Received Nov. 28, 2017; accepted Aug. 29, 2018) 